\title{
Call for Special Issue Papers: Tissue Engineering: Part A- Special Issue on Oncology and Tissue Engineering
}

\section{Deadline for Manuscript Submission: November 30, 2020}

\author{
Guest Editors: Prof. Tzu-Wei Wang; National Tsing Hua University, Taiwan; \\ Prof. Yadong Wang, Cornell University, USA.
}

\section{General Information}

Tumor cells reside in complex microenvironments in which they are subjected to a variety of physical, chemical, and biological stimuli that influence cell behavior and ultimately the progression and maintenance of the tumor. The multi-dimensional structure, organization, and complex relationships in diverse tumor microenvironments make cancer hard to be treated and easily recurrence. Tissue microenvironment has been extensively studied in many areas of tissue engineering and it plays a crucial role in cell signaling and regulation of normal and malignant cell functions.

Unlike 2D monolayer culture model, 3D models of solid tumors closely resemble in vivo tumor microenvironment and metabolic characteristics. The cell-cell and cell-matrix interplay are in line with more mimicking the tumor behavior in vivo. It is important to use three-dimensional constructs based on tissue engineering principles to understand cancer cell - normal cell interactions, and how cancer cells actually grow, and how cancer cells go into the bloodstream and metastasize to other tissues.

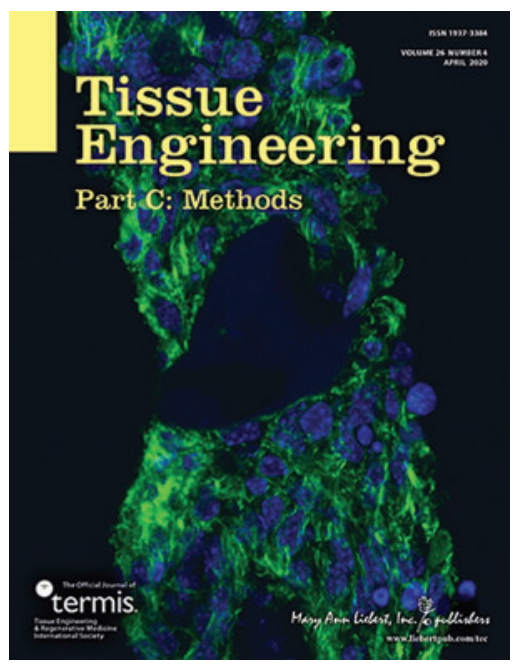

By gaining a better understanding of the tumor microenvironment that governs tumor dynamics, we can make advances in combatting cancer dormancy, recurrence, and metastasis. These models and platforms have the potential to investigate the suitable biomaterials, culture conditions, and material structure to control tumor growth and spread.

The collaborative works between tissue engineering and cancer biology will be promising for creating novel experimental models that mimic cancer pathophysiology to elucidate specific cancer phenomena that are otherwise difficult to examine in vivo. Taken together, these considerations will enhance the relevance of in vitro tumor models, biological understanding, and accelerate treatment exploration ultimately leading to improved clinical outcomes and diagnosis in cancer.

Topics of interest for this special issue include but are not limited to:

- Smart 3D hydrogel to study tumor formation, invasion, and cancer progression

- The role of extracellular matrix property in development of tumors

- 3D organoid/organotypic models for cancer tissue engineering

- Biomimetic tissue-engineered technologies and systems for cancer research

- New technology or methods developed to model the behavior of tumor cells or anticancer drug response

- Modulating physiochemical properties of the scaffold to study tumor growth and metastasis

- Tissue-engineered nanomedicines for cancer

Mary Ann Liebert, Inc., publishers, 140 Huguenot Street, New Rochelle, NY 10801, USA. 
We also encourage submission of papers that have been published in conference proceedings, with the requirement that the authors have made significant extensions as compared to the already published version of the study. For more information, visit the Instructions for Authors at www.liebertpub.com/ten

Manuscript Submission Deadline: November 30, 2020

If you are interested in submitting a manuscript for the special issue, but need extra time because of COVID-19, please kindly notify the guest editors in advance. We'll be happy to work with you.

Questions or pre-submission queries? Please contact Guest Editors Professor Tzu-Wei Wang at twwang@ mx.nthu.edu.tw; Professor Yadong Wang at yw839@cornell.edu

\section{Visit the Instructions for Authors:}

www.liebertpub.com/ten

\section{Submit your paper for peer review online:}

https://mc.manuscriptcentral.com/ten 